\title{
Subjective perception of natural scenes: the role of color
}

\author{
Nadia Bianchi-Berthouze \\ Database Systems Lab, University of Aizu, Aizu Wakamatsu, Japan \\ e-mail: nadia@u-aizu.ac.jp \\ http://www.u-aizu.ac.jp/ nadia
}

\begin{abstract}
The subjective perception of colors has been extensively studied, with a focus on single colors or on combinations of a few colors. Not much has been done, however, to understand the subjective perception of colors in other contexts, where color is not a single feature. This is what the Kansei community in Japan has set itself to, by exploring subjective experiences of perceptions, and colors in particular, given its obvious influence on humans' emotional changes. The motivation is to create computational models of user visual perceptions, so that computers can be endowed with the ability to personalize visual aspects of their computational task, according to their user. Such a capability is hypothesized to be very important in fields such as printing, information search, design support, advertisement, etc. In this paper, we present our experimental results in the study of color as a contextual feature of images, rather than in isolation. The experiments aim at understanding the mechanisms linked to the personal perception of colors in complex images, and to understand the formation of color categories when labeling experiences related to color perception.
\end{abstract}

Keywords: Visual Subjective Impression, Dynamic User Modeling, User Externalization, Color Perception, Kansei Engineering

\section{INTRODUCTION}

Color is defined as "that characteristic of a visible object or light source by which an observer may distinguish differences between two structure-free fields of the same size and shape, such as may be caused by differences in the spectral composition of the light concerned in the observation" [1]. But, as Sir Isaac Newton said "Rays are not colored" [2]. Spectral power distribution exists in the physical world, but color exists only in the eye and the brain. In other words, color is an inherently subjective experience.

As such, color perception has been extensively studied. Of course, physiological studies have investigated the mechanisms responsible for so-called visual perception of colors [3,4,5]. Such studies result in findings in the psychophysics of perception, with the identification of the different pathways (visual cortex, etc.). From a computational point of view, such theories have sometimes been applied to the implementation of computational and robotic systems $[6,7,8]$.

The psychology of color perception has been investigated as well $[9,10,11]$. From our perspective, however, the focus of these studies has been primarily on the particular role of one or of a combination of a few colors with respect to a task or a given emotion.

Finally, there have been cognitive science studies with categorical perception as a mean to explain color segmentation. Overall, these studies do tend to separate color from other components of perception and the subjective aspects are not really being investigated. This is very much the case in computational linguistics, with studies investigating color category formation and its relation with languages and cultures $[12,13,14,15,16,17]$. The influence of social interaction on the process has also been discussed [18]. This interesting study shows the emergence of color categories in a population of agents playing color-related language games. Yet, this study lacks a context that could shape the emergence of certain categories of color versus others.

This is what the Kansei community in Japan has set itself to, by exploring subjective experiences of perceptions, and colors in particular, given their obvious influence on humans' emotional changes. In Kansei research, the main point is to observe and characterize the personal experience of each individual $[19,20,21,22,23,24,25,27,28]$. The motivation is to create computational models of user visual perceptions, so that computers can be endowed with the ability to 
personalize visual aspects of their computational task, according to their user. Such a capability is hypothesized to be very important in areas such as printing, information search, design support, advertisement, etc.

Image retrieval, for example, has been addressed by using keywords, textual descriptions and similarity criteria $[29,30,31,32]$. To account for the way humans look for information, however, an additional retrieving modality is necessary, namely, one modality that relates to the affective content carried by the image. Indeed, it is that affective content that will often explain why a person will select a particular image from a pool of many others satisfying the same objective criteria (e.g., the percentage of a certain color, the type of objects). In some applications such as design or advertisement for example, affective content will be the only criterion for selection.

In our research $[28,33,34]$, we have been investigating how to endow a computer with the ability to process and treat affective (and therefore subjective) criteria. In this paper, we will discuss the results of some experiments we have been carrying out to characterize subjective visual experiences and the relevance of color features over others. We will conclude by describing a methodology to create adaptive computational user models, based on the mapping of lowlevel features of images onto impression words labeling the conveyed impression.
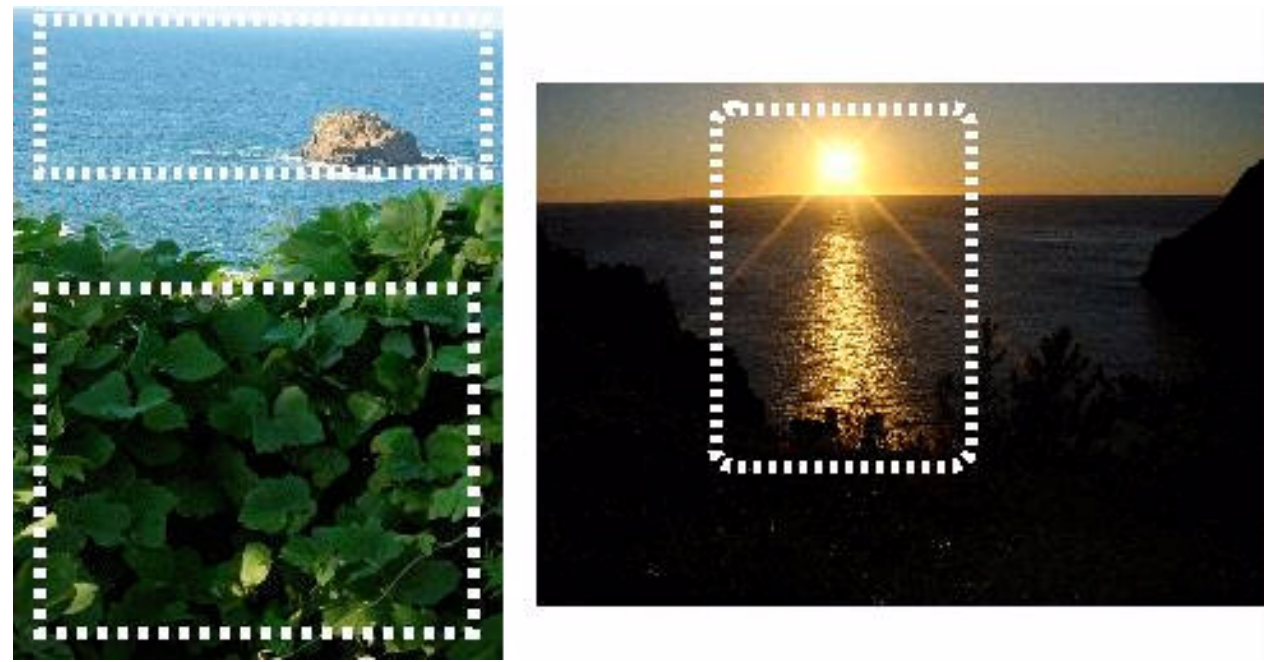

Figure 1: Our brain uses attention and selection mechanisms to interpret images. The impression conveyed by these images can change according to the focus of attention of the user.

\section{SUBJECTIVE VISUAL EXPERIENCE: SOME KEY ASPECTS}

We performed experiments, aimed at identifying key characteristics of image perception and its subsequent subjective experience. Our experiments have not been designed to help clarifying the neurophysiological mechanisms underlying subjective experiences. Instead, our focus has been to find a suitable abstraction level, whereby subjects are conscious of their subjective experiences and communicate them.

Our approach is somewhat similar to Munsell's description of color space as dimensions of hue, intensity, and saturation seen in the CIE chromaticity space [35]. The geometrical dimensions of that space have been tailored to match the properties of the subjective experience of color as measured psychophysically, and expressed in terms that are agnostic to any particular neurophysiological theory of color representation but that are close to the way humans use to describe their color experiences [36].

Other researchers have made similar attempts. Well known to the community is Kato's studies [37] in which subjects were asked to observe a set of impressionist paintings, and to label them by using a predefined set of impression words. The intensity of the impression was evaluated on a graded scale. The results were used to create a statistical model of the mapping between images and impressions. By using multivariate analysis, the authors could describe the distribution of impression words with respect to dimensions extracted from the images low-level features. This study, 
however, did not attempt to elicit which of these dimensions were salient to the impression. In fact, improvements of the model are only considered via a simple relevance feedback, which has been shown to be relative and not sufficient to improve the quality of such a complex mapping [38].

While an image being observed is complex information per se, the interference of various other factors such as past experience, state of mind, goal orientation, etc., can lead to highly variable readings of the same information. For example, impressions arising from the observation of a landscape do not only derive from its visual characteristics, e.g., color, spatial organization, but also from past experiences the brain recalls when stimulated with those characteristics. Because our visual system uses selection and attention to filter the information from the observed object to the brain [39], there is no telling which particular emotive state will be experienced when stimulated by a same stimulus over time. In fact, state of mind, goal and/or past experience direct attention on some aspects of an image and at different levels of abstraction [40]. For example, different areas of the sea landscape shown in Figure 1 (left) convey different impressions: the blue sea might convey an impression of freedom while the dark wall of leaves may convey the opposite impression. In the second picture of the same figure, a romantic impression is caused by degrading nuances of yellow hues on a dark background, while a peaceful impression is caused by the slight illumination of dark regions, regardless of its hues.

One more point should be considered. In these experiments, natural language is chosen as a mean of communication because, among all channels, language is consciously controlled and is a powerful tool that we exploit intensively. However, as rich as it might be, natural language does not have a one-to-one relation with subjective impressions. Words are associated with a definition given in the dictionary, but these definitions can be very fuzzy, especially with respect to subjective states. A same word may be used to convey different nuances of a same subjective impression, or be used in a metaphorical way. Even the way in which we refer to our impressions can change because our language ability, e.g., the richness of our lexicon, varies over time as well as our ability to discern between visual experiences. In the early stages, a child will only be attracted by bright colors but will subsequently learn to appreciate finer differences in her/his visual experiences [11,41].

\begin{tabular}{|l|c|c|c|c|c|c|c|c|c|c|}
\hline & \multicolumn{5}{|c|}{ Male Subjects } & \multicolumn{5}{c|}{ Female Subjects } \\
\hline \multicolumn{1}{|c|}{ Words } & S-1 & S-2 & S-3 & S-4 & S-5 & S-1 & S-2 & S-3 & S-4 & S-5 \\
\hline Atsui (hot) & $86 \%$ & $80 \%$ & $96 \%$ & $94 \%$ & $90 \%$ & $88 \%$ & $10 \%$ & $94 \%$ & $80 \%$ & $73 \%$ \\
\hline $\begin{array}{l}\text { Bukimina } \\
\text { (uncanny) }\end{array}$ & $98 \%$ & $86 \%$ & $100 \%$ & $20 \%$ & $98 \%$ & $94 \%$ & $60 \%$ & $20 \%$ & $46 \%$ & $40 \%$ \\
\hline Kakkoii (cool) & $46 \%$ & $96 \%$ & $96 \%$ & $50 \%$ & $80 \%$ & $86 \%$ & $72 \%$ & $50 \%$ & $86 \%$ & $96 \%$ \\
\hline $\begin{array}{l}\text { Kimotiwarui } \\
\text { (weird) }\end{array}$ & $88 \%$ & $96 \%$ & $98 \%$ & $43 \%$ & $86 \%$ & $96 \%$ & $40 \%$ & $43 \%$ & $82 \%$ & $32 \%$ \\
\hline Kowai (fearful) & $60 \%$ & $78 \%$ & $80 \%$ & $38 \%$ & $84 \%$ & $92 \%$ & $66 \%$ & $38 \%$ & $56 \%$ & $98 \%$ \\
\hline $\begin{array}{l}\text { Miwakuteki } \\
\text { (enchanting) }\end{array}$ & $92 \%$ & $92 \%$ & $98 \%$ & $48 \%$ & $98 \%$ & $92 \%$ & $28 \%$ & $48 \%$ & $90 \%$ & $86 \%$ \\
\hline Osorosii (horrible) & $98 \%$ & $80 \%$ & $78 \%$ & $58 \%$ & $70 \%$ & $96 \%$ & $92 \%$ & $58 \%$ & $76 \%$ & $98 \%$ \\
\hline Sabishi (lonely) & $96 \%$ & $80 \%$ & $94 \%$ & $44 \%$ & $100 \%$ & $86 \%$ & $12 \%$ & $40 \%$ & $84 \%$ & $72 \%$ \\
\hline Shizuka (quiet) & $76 \%$ & $70 \%$ & $82 \%$ & $40 \%$ & $78 \%$ & $80 \%$ & $24 \%$ & $40 \%$ & $92 \%$ & $96 \%$ \\
\hline Tsumetai (cold) & $88 \%$ & $88 \%$ & $88 \%$ & $36 \%$ & $96 \%$ & $84 \%$ & $48 \%$ & $36 \%$ & $96 \%$ & $92 \%$ \\
\hline
\end{tabular}

Table 1: The table shows the subjects' variability in labeling the visual impression of a same set of images on 2 subsequent classification sessions. Each number indicates the percentage of images that have been classified differently in the 2 sessions by a same subject S-\#. 
Our research is an attempt to overcome the problems discussed above, by deepening the understanding of the issues involved in subjective perceptions. In our experiments, the subjects are involved in reconstructing the cognitive pathways underlying their subjective experiences. In this paper, we report the key aspects we have identified relative to users' subjective visual experiences: variability of the subjective experiences, context dependence, language generalization and externalization of user naive models [42]. These characteristics will help us define the requirements for the Kansei User Modeling process.

\subsection{Variability of visual impressions}

Our first set of experiments aimed at evaluating the variability of users' subjective experiences. The subjects were 10 Japanese university students. To select an appropriate kansei lexicon (i.e., impression words), the subjects were initially asked to classify 25 landscape images by using a free selection of impression words (e.g. quietness, loneliness, etc.). Among the resulting words, 10 were selected. The selection was based on the frequency of use within all subjects, and within each single subject. Words, whose meanings were only describing the low-level features of the images, e.g., "dark"', were discarded, no matter their frequency. In subsequent experimental sessions, the subjects were asked to classify the visual impressions conveyed by single images, using the previously selected impression words. In each experiment, the number of images was increased and the order of presentation was changed and interleaved with new images. Each session was separated by a month period.

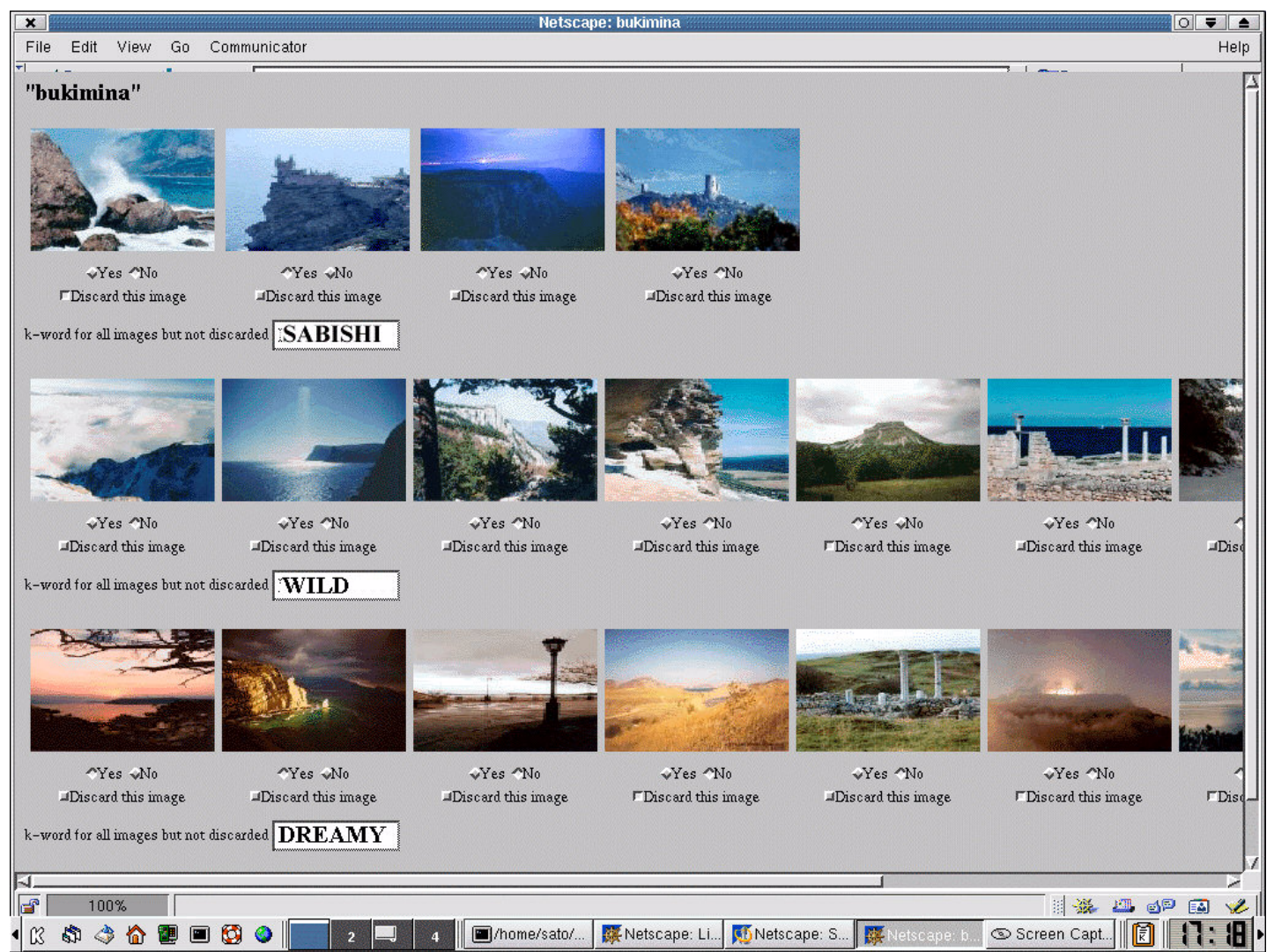

Figure 2. The reclassification of the set of images classified at first as conveying a same impression of "bukimina" (uncanny). The aim of the experiment is to better characterize an impression by using more specifics words.

While all subjects showed high consistency in classifying images as not conveying a certain visual impression, they did show high variability in classifying images as conveying a certain impression. Table 1 shows the variability over 2 sessions of 50 images. The first column shows the Japanese words used by the subjects to classify the images. The results obtained by each subject are reported in the next 10 columns (one column per subject). The number indicates the 
percentage of images classified, in a first session, as conveying the impression indicated in the left column, and then reclassified, in the second session, as not conveying that impression. The variability is very high with an average value of $73.2 \%$ and a standard deviation of $24.2 \%$. These results confirm our hypothesis: namely, that an image is a complex set of information, and that its perception, via selection and attention, is affected by factors that vary over time, e.g., state of mind, goal, etc.

\subsection{Visual impression naming and context dependencies}

Another set of experiments was carried out to model nuances of impression words. During interactive sessions, subjects were asked to go through the set of images they had previously classified as conveying a same visual impression, and to arrange them in sub-groups according to nuances of that impression. If images were not thought to convey the initial impression, they could be discarded.

The experiments showed a high correlation between task performance and lexical abilities of the subjects. Limited vocabulary (even in the native language) resulted in the subjects reporting great difficulties in assigning new labels to each sub-group of images. While this may have been an artifact resulting from the selection of subjects available, we hypothesize that it is, in fact, a general phenomenon and that it should further be investigated. As a supportive evidence, it was observed that when an additional set of impression words was provided to the subjects, all of them could carry out the experiments. With the specialization of the vocabulary, inconsistencies between low-level features of images belonging to a same impression word could be solved. Apparent inconsistencies were accounted for by a tendency to use the same impression word to identify a large range of impressions. For example, "quietness" was used to indicate both a peaceful feeling and a sense of loneliness.

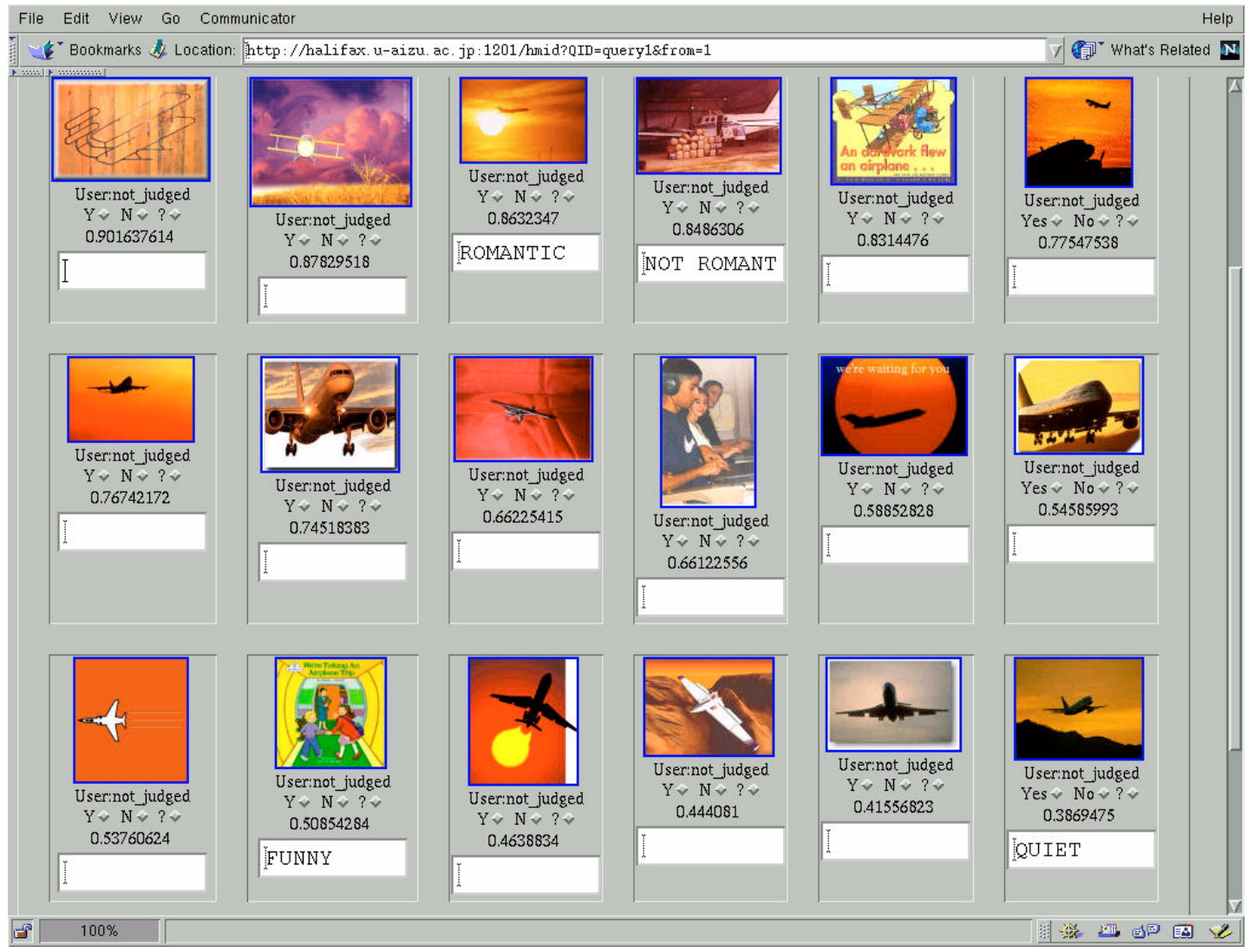

Figure 3. This screenshot shows the result of an automatic retrieval session performed by a web-based kansei search engine [27] upon the query "romantic images of airplane" entered by a user. It displays the best 18 hits for "romantic" after the filtering of 452 images of airplanes. The user can input relevance feedback by writing impression words under each retrieved image in order to improve the result of the automatic search. 
Two additional observations were made. First, the fact that images were shown simultaneously increased user judgment variability, as well as the number of images discarded. This result highlights the importance of "comparison" (similarity) and "context" as powerful tools used by subjects in their judgement. Secondly, the context was also shown to relate to the category of images being considered and not only to the context in which images were observed. Experiments on different classes of images confirmed our hypothesis that the context (i.e., high-level image content and goal of the user) affects the mapping between the low-level features of an image and the subsequent visual impression. The mapping of "quietness" into low-level image features vary according to the class of images, i.e., different low-level features between quiet outdoor landscapes and quiet interiors.

\subsection{Features saliency}

We attempted to automatically identify the saliency of color over other low-level features, in identifying nuances of a given impression word. The images classified by each subjects during the different experiments were divided into two sets. A first set was used as a training set to create computational models of impression words for each user. These models were created by using clustering and neural network algorithms, such as described in section 3.1.

To evaluate the feasibility and effectiveness of the models, we selected a real-world application in which the computer system has to handle requests involving subjective information. Nowadays, many web sites (e.g., Hallmarks, BlueMountain) have been set up for users to send virtual postcards to friends/relatives on various occasions such as birthday, dates, national holidays, etc. Typically, these sites provide a collection of images for each category of card (a virtual postcard will be an image, a layout, a song, and a text entered by the user). It is therefore quite unflexible and limited. It takes into account the sensibility of the user in so far as the user finds an item of his/her liking, by browsing systematically the collection. When unsuccessful, the user will either have to switch to another site, or find a best compromise. On the other hand, many search-engines are available on the Internet for retrieving images, e.g., Alt $\mathrm{I}$ ista [43], google [44, yahoo [4 5].
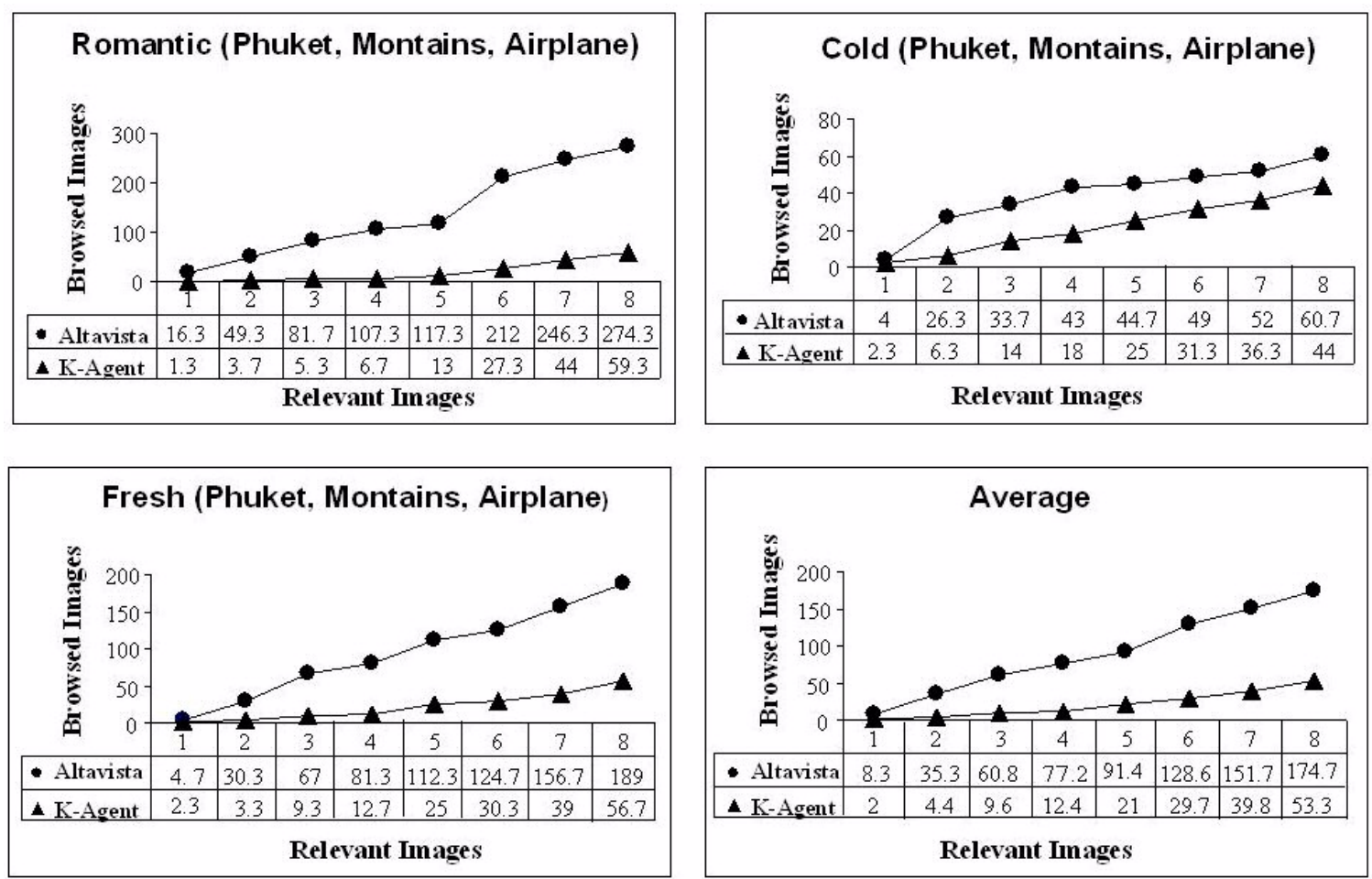

Figure 4. Comparative evaluation of the retrieving process in AltaVista and filtering based system. 
Thus, the following scenario was considered: users were asked to retrieve images for a virtual postcard, according to (1) an objective keyword; and (2) a visual impression to be conveyed by the postcard, e.g., retrieving a romantic image of airplanes (see figure 3). Users were asked to perform that task using (1) their usual WEB-based search engine, i.e., querying the search engine using the objective keyword and then manually filtering against the subjective keyword; and (2) our own application (see implementation details in [27]) with the constructed models filtering the retrieved results.

Figure 4 shows a graphical representation of the retrieving process, comparing the performance of the user manually browsing the results of AltaVista, and the result of our system. The horizontal axis denotes the number of images retrieved, while the vertical axis indicates the number of images browsed. In effect, the graph represents the so-called "recall" for the two cases.

In all instances, superior performance is obtained when filters are used, and the time required for the user to retrieve images is reduced very significantly. A notable exception is the impression word "cold". While the filters still outperform AltaVista, the difference in performance is not as significant as in the other impression words. The better performance of AltaVista for this particular word could be accounted for by the large presence of images conveying the impression "cold" in its collection. Indeed, because AltaVista doesn't order its images with respect to a given impression, its particular performance can only be marginal.

Because of the filters' performance, users were more incline to engage in a larger search than when they had to do it manually. For example, a raw query of AltaVista on a set of keywords such as "Phuket", "Mountains" or "Airplanes" yielded thousands of images. In this experiment, we evaluated that in average, a user had to browse up to 8 images before finding a satisfactory one. Typically, for the user to find 2 suitable images, s/he had to browse through 35 images (4 pages) in AltaVista while only 4 images were needed with the filter based system.

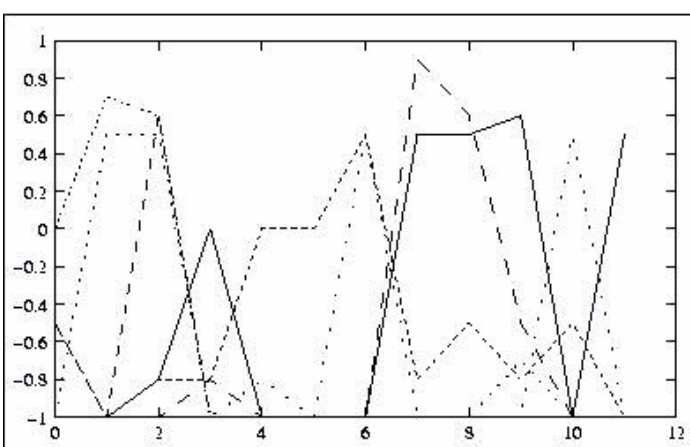

a) Lonely nuance: hue features

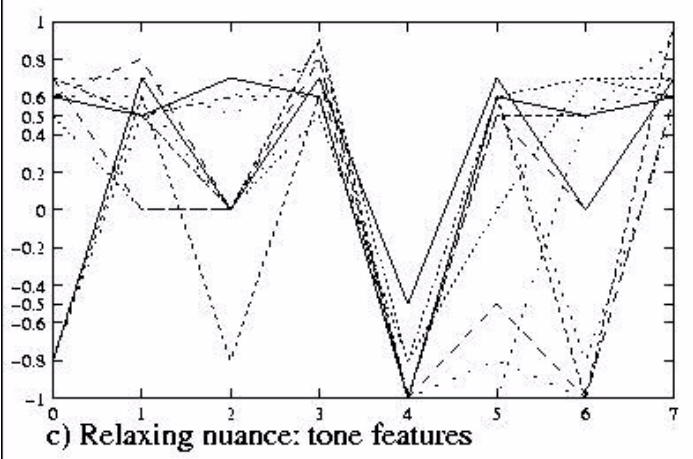

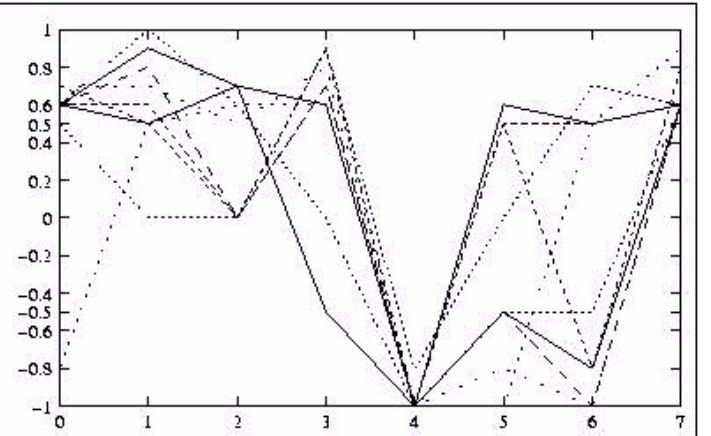

b) Lonely nuance: tone features

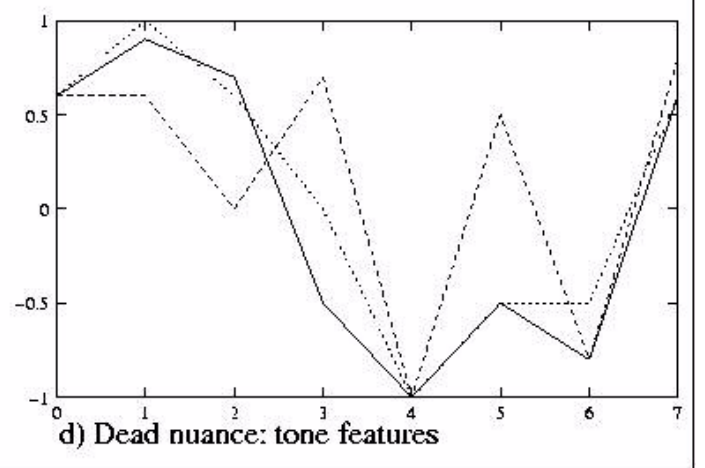

Figure 5. The figure shows the distribution of color features on nuances of the word "quiet'. Each graph shows the distribution of the low level features for the set of images reclassified using a same nuance a) hue for the nuance "lonely"; b) tonality for "lonely"; c) tonality for "relaxing ; d) tonality for "dead". We observe that tonality features seem to discriminate better the nuances of the word "quiet" than the hue features do. 
The solid performance of the models using only color features showed the predominance of color over other features, such as texture and shape, in discriminating a large set of impressions. However, it was also shown that for some impressions, such as "imposing", color was not the primary low-level feature and the filter's performance was lower.

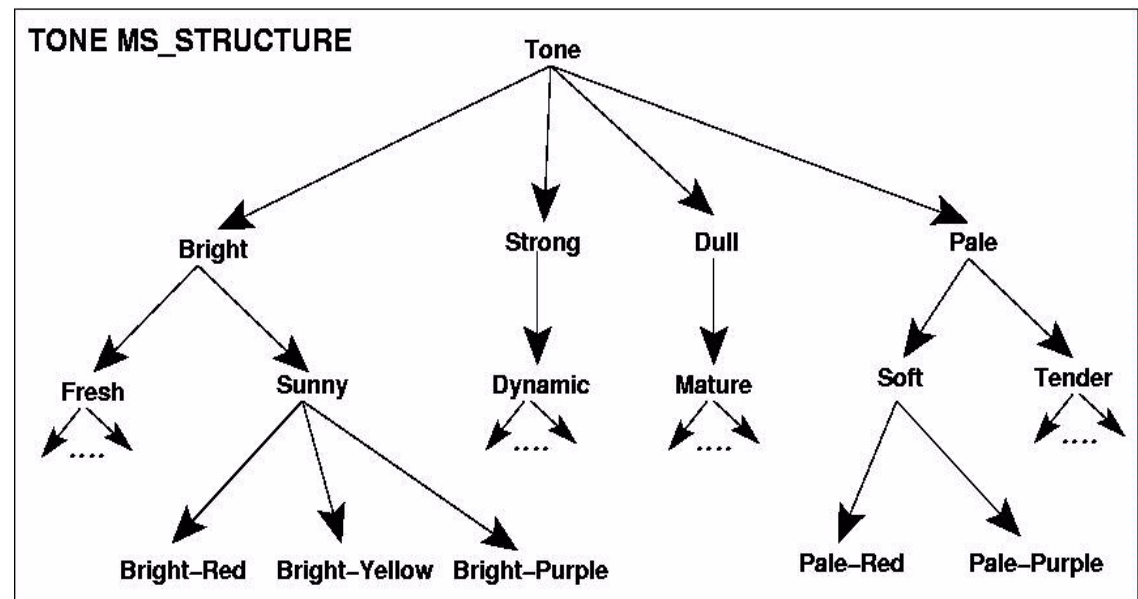

Figure 6. Hierarchical aggregation of color features into higher-level tonality concepts.
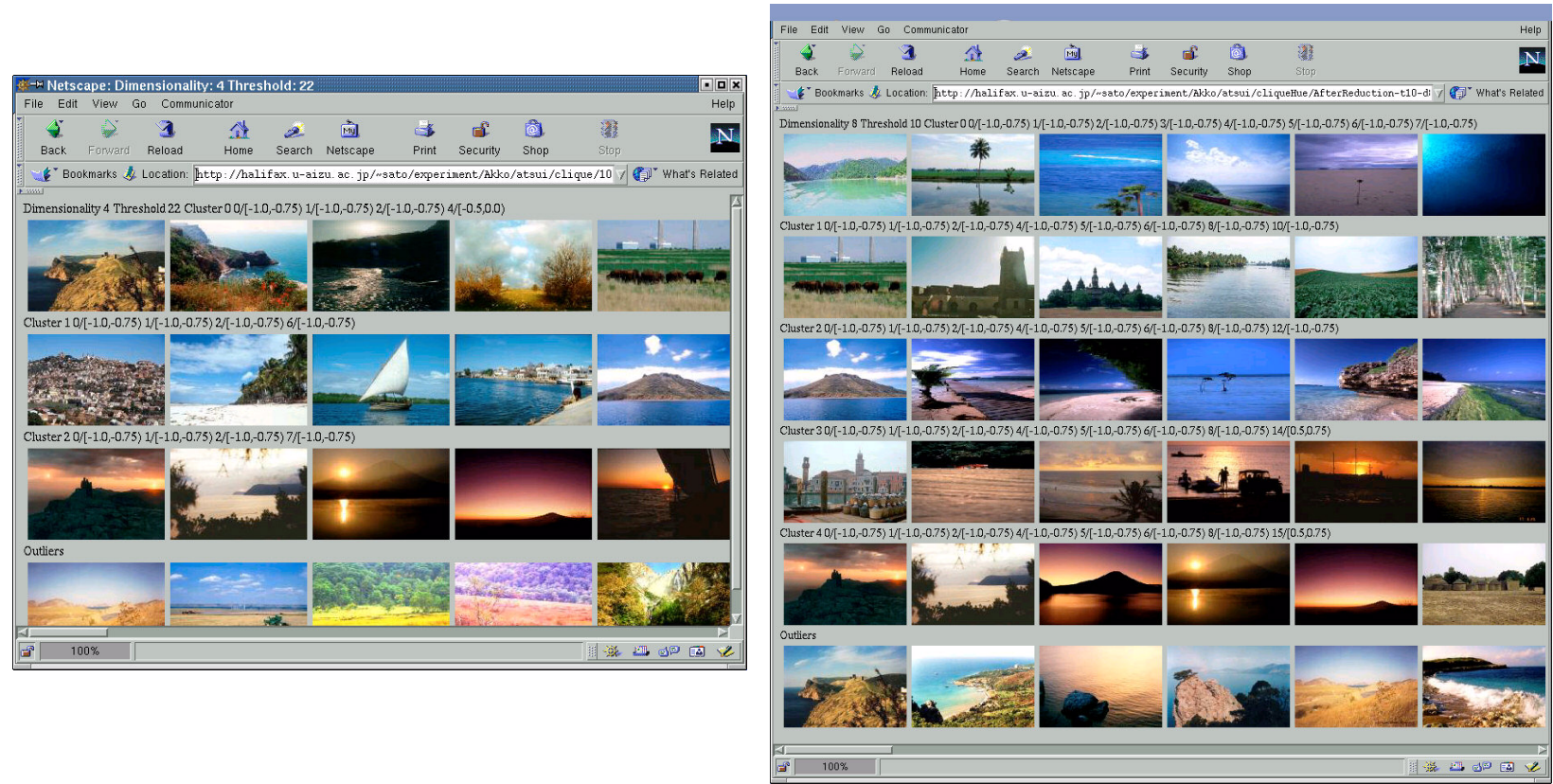

Figure 7. Results of the clustering performed on 2 different aggregation level of features of the same set of "quiet" images. The clustering aims at identifying groups of images that can be associated with nuances of the impression word "quiet".

We also examined which color characteristics were relevant to a visual impression. Figure 5 shows the distribution of the set of "quiet" images with respect to hue and tonality features. In the first graph (top left), the horizontal axis represents 6 hues (from red to purple) while, in the remaining graphs, it represents the 8 tonalities of color (black, grey, 
dark grey, white, dull, pale, bright, strong). The vertical axis represents the percentage of each hue or tonality features in an image. Each image is thus represented by a polyline. The upper graphs describe the distribution of the color features for the images conveying a "quiet-lonely" impression while the third and fourth graphs represent the distribution of images conveying the impression "quiet-relaxing" and "quiet-dead", respectively. It can be observed that tonality features are more appropriate than hue features to discriminate between nuances of the word "quiet".

These graphs also illustrate the role of another factor: the level of description of the modelled features. To analyze and capture the relevant features, we suggest considering the low-level features as leaves of hierarchical meta-schemas (see Figure 6). These meta-schemas support the creation and management of various interpretations of the low-level features of an image. We performed an automatic clustering of the images by using models built on different aggregation levels of the color features. Figure 7 shows the result of the clustering process for two different set of features: right, when low-level aggregation is used, i.e., the HSI space is decomposed into 28 regions (combination of 6 hues and 4 tonality features plus 4 grey tones); left, when only tonality space is considered. For a given impression word, the left clustering shows a better fit to the clustering performed by the user. Still, further studies will be necessary to understand how to select the relevant abstraction level of description for each impression word.

\subsection{Externalization Process}

As far as the personalization of user models is concerned, relevance feedback [29,30,31,32] has been the main modality of interaction considered between system and user. However, our own experiments have shown the limits of relevance feedback, in particular, its relative meaning and its inconsistency over time. To overcome these limitations, we have explored the feasibility of having the user actively participate to the adaptation process of the user model. This is an important requirement for dealing with the issue of multiple readings of the same image. To this end, we have looked at the possible value of triggering externalization processes in the subjects so that the focus of attention and the selected features could be detected. Externalization processes typically help users in being aware of the cognitive processes behind their subjective experience [42].

We performed a set of experiments were the subjects were asked to describe the reason of the impression that each image was conveying. In the experiments, we aimed at leading the subjects to express their hypotheses in terms of lowlevel features of the images. One could argue that it is almost impossible for a user to determine the real factors/dimensions that give rise to an impression. We acknowledge the difficulties of such process and the uncertainty of its results. However, while this process is difficult, it has been shown [46,47], through empirical results, that subjects can be trained to become sensitive in perceiving dimensions. Secondly, and very importantly, the position we take in this work is to consider the externalisation process primarily as a support to the modeling activity so that the complexity of the data to be analyzed is reduced.

Our preliminary experiments showed the difficulties, but also the feasibility, of such an approach. As a result of these experiments, we have identified some requirements for an effective participation of the subjects. In particular we identified four main mechanisms to trigger externalization processes: inconsistency detection, similarity measurement, word specialization and visual representation of externalized cognitive pathways.

\section{CREATING COMPUTATIONAL MODELS}

Our methodology combines two processes: 1) an automatic modeling of the mapping between image features and impression words; and 2) an interactive refinement of the model via externalization processes. The first process is achieved by a learning kernel module. The second process makes use of a conceptual space where cognitive maps, resulting from the externalization process, are dynamically built to support the interaction between the user and the modeling process. In the next sub-section, we briefly describe the two modules. A more detailed description can be found in $[28,33,34]$.

\subsection{The learning kernel}

The rationale behind the specification of the learning kernel is that the "meaning" of an impression word is partially grounded into the low-level characteristics of the images conveying that impression. The learning kernel is thus set to learn associations between perceptual states and impression words under user supervision. A perceptual state is a signature of an image, extracted using a set of image processing algorithms. Such a signature describes the image in terms of color, texture and shape features. 
The learning kernel contains a set of word modules, each associated with an impression word. A word module consists of a set of neural networks, which learn the relations between a subset of the perceptual state and the impression word. A saliency factor, or weight, is associated with each neural network and updated through user feedback. With this mechanism, the word module can deal with different subjective impression words relating to different categories of perceptual characteristics. For example, "romantic" or "fresh" might relate to color features, whereas "imposing" or "brave" might relate to shape characteristics.

The neural networks are of the 3-layers forward type, trained by back-propagation with momentum. Unlike in typical applications, where learning sets are constructed off-line, we are dealing with a continually evolving training set (a byproduct of the user feedback). To limit the size of the training set, an automatic analysis is performed before adaptation. Representatives for the training set are selected through a stratified sampling, using multidimensional clustering algorithm. The stratified sampling is based on the concept of impression words specialization [40].

Since the lexicon is expected to evolve over time, as the interaction unfolds (i.e., as new words are introduced), each word is independently dealt with by a word module in charge of learning its particular associations with perceptual states. Adding a new impression word consists of initializing a new word module, and constructing a training set. New words can be added at run-time as users utilize them to enter feedback on a retrieved image. The modules are connected into a network that indicates relations between impression words. Possible relations are "opposite", "synonym", "related" and "nuance".

\subsection{A conceptual space to support externalization processes}

To overcome the limit of the relevance feedback, we propose a conceptual space [48] as a tool to support the formulation by a user of hypotheses and the translation of these hypotheses into formal expressions that can be tested and integrated into the computational models. A conceptual space is based on concepts (in our case, visual impression concepts). A concept is defined by a set of dimensions from different domains or categories. The description of a concept is not unique but context dependent. A domain corresponds to the perceptual dimension(s) by which the system and the user perceive the image (e.g. color). A domain has a geometrical and topological structure that supports the definition of relations among its dimensions, e.g., similarity. As discussed in section 2.2, similarity is an important mechanism in cognition. Often, when making a subjective/objective experience, we are brought to relate it to previous experiences and in particular to evaluate how similar or different they are.

The set of concepts is not defined a priori. Low-level dimensions are defined according to image processing algorithms and tuned following user feedback. High-level dimensions are defined as a combination of lower level dimensions. This hierarchical structure is constructed through the modeling activities and users' externalisation processes. For example, the meaning of the low-level categories "color" and "tone" can be associated to the Munsell structure [35] with their dimensions formally specified as a segmentation of the Hue-Saturation-Brightness space. The high-level category "sad" can be described in terms of "dark" or "greyish" dimensions defined respectively in the "tone" and "intensity" categories.

Externalisation processes are rarely self-triggered. The role of the system is therefore to lead the user into this difficult process. This system activity is implemented in the form of objective representations of the externalisation steps, combined with the evaluation of the user's hypotheses. Such representation is called multimedia cognitive map $[49,50,51]$ and provides a simple yet intuitive mean of highlighting important strands of thoughts.

Naturally, the resulting cognitive map does not represent the entire belief system but creates an objective ground for reasoning. The multimedia cognitive map contains user feedback, user hypotheses and inconsistencies identified by the modeling system. By using multimedia cognitive maps, a user can easily recollect the path of his/her externalisation process and correct or complete his/her hypotheses. The creation/evaluation of a multimedia cognitive map takes the form of a cyclic loop, identifying inconsistencies that help the user to correct or complete his/her naïve mental model. We identify the following types of inconsistencies: a) intrinsic or derived from a different evaluation of the same selected information (temporal evolution in the observer); b) true or derived from the misuse of a word in conveying a subjective experience and c) attentional or derived from a different reading of the information by the selection mechanism.

The detection of intrinsic inconsistencies reveals a training set that is not anymore a correct or extensive sample for the associated impression word. It helps the user to identify more meaningful examples for the learning process. The detection of true inconsistencies can lead the user to discard specific samples from the training set and/or to identify a 
more appropriate impression word or nuance for the classification of the images. Finally, the detection of attentional inconsistencies signals a limited or incorrect low-level characterisation of the image, i.e. an incorrect selection of features to be associated with an impression word.

\section{CONCLUSION}

In this paper, we reported our experimental studies to validate the working hypothesis that the visual impressions conveyed by an image (here, a landscape image) can be derived from its color features. Validating this hypothesis is an important issue as it would open the way for computer systems to be involved in new application fields where affective processing of information is necessary, for example, design support, entertainment, advertisement, etc. In our experiments, color is explored as one of many dimensions of a subjective visual experience. Indeed, it is our premise that color should not be studied in isolation. We reported the results of psychological studies exploring the relative importance of color and other low-level features in terms of context, as well as results from communication studies in which subjects were asked to label image-related experiences. The results showed the high variability that characterize both the subjective visual experience and its externalization through natural language.

The success obtained in image retrieval sessions performed by using computational models based on color features only, suggested that visual impressions rely strongly on color features. However, it was also shown that some categories of visual impressions cannot be suitably learned from color features only and do require the combination of color with other features. At this stage of our research, this issue is still open.

Overall, our studies have brought a positive indication that extracting visual impressions from low-level features is feasible. They have also helped us identify guidelines for designing personal computational models. In particular, they suggested a two-phase methodology to overcome the limits of conventional automatic modeling approaches. In those approaches, personalization of the user model is generally based on relevance feedback only. Our experiments have shown, however, that this type of feedback has only relative value and, in addition, is very often inconsistent over time.

As an alternative, we have suggested that the user should actively participate in the adaptation process of the user model. To this end, we have identified some key requirements for such effective participation to take place, and also for filling the semantic gap between user interpretation and image low-level features. Indeed, the user is generally not aware of the reasons underlying his/her feedback, i.e., why an image does or not convey a certain impression. However, we showed that if his/her mental processes were triggered by using inconsistencies and similarity detection mechanisms, then the user could formulate hypotheses, even low-level ones.

Naturally, an important correlate of such observation (with respect to human-machine interaction) is that these hypotheses have to be translated into a form that could be handled by the system. Thus, it is necessary to create a support for hypotheses formalization that can be understood by both the user and the system. Consequently, we have proposed a conceptual framework in which subjective high-level (as well as low-level) concepts, such as color, are topologically and geometrically defined, and adapted over time. The objective characterization is necessary to quantify the structural features of the subjective experience in objective terms so that the user's hypotheses can be formalized, tested and evaluated. For example, the concept "red" can be objectively used to describe a well precise range of color. Such range, however, is not statically predefined but can be adapted over time, by the user or by the system. This objective definition of concepts makes it possible to establish relations between them, relations which are at the basis of the human mental processes involved in judgement. We believe that the use of conceptual spaces will also prove useful to extend the study of color categories to areas other than computational modeling, for example, color naming in linguistics.

\section{REFERENCES}

1. G. Wyszecki, W.S. Stiles, Color Science: Concepts and Methods, Quantitative Data and Formulae, Wiley, New York, 1982

2. I. Newton, The First Book of Opticks: or A treatise of the reflexions, refractions, inflexions and colours of light, Walford, London, 1704

3. S. Zeki, A vision of the brain. Oxford, England: Blackwell Scientific Publications, 1983

4. D. Hubel, Eye, Brain, and Vision, Scientific American Library, series \#22, New York. 1988. 
5. S.A. Engel, C.S. Furmanski, "Selective Adaptation to Color Contrast in Human Primary Visual Cortex, Journal of Neuroscience, 21 (11), pp. 3949-3954, 2001.

6. F. C. Wanderley, M. H. Fisher, "Multiscale Color Invariants Based on the Human Visual System Juliana", IEEE Transaction on Image Processing, 1011), pp. 1630-1638, 2001

7. M. D. Zmura and G. Iverson, "Color constancy. I. Basic theory of two-stage linear recovery of spectral descriptions for lights and surfaces", Journal of Optic Society of American Association, 10 (10), pp. 2148-2165, 1993

8. L. Tao and G. Xu, "Color in machine vision and its application", Chinese Science Bulletin, 46 (17), pp. 1411-1421, 2001.

9. F. Birren, Color and Human Response, Wiley, John \& Sons, Incorporated, 1978.

10. Readings on color, A. Byrne, D.R. Hilbert (Eds), 1-2, MIT Press, Cambridge Massachusetts, 1997

11. S. Kobayashi, Colorist: a practical handbook for personal and professional use, Kodansha, 2000

12. J. M. Lammens. A Computational Model of Color Perception and Color Naming. PhD Thesis, State University of New York at Buffalo, 1994

13. E. Rosch, "Universals in color naming and memory", Journal of Experimental Psychology, 93, pp. 10-20, 1972.

14. B.L. Whorf. Language, Thought and Reality, The MIT Press, Cambridge, MA, 1956.

15. B. Berlin, P. Kay, Basic Color Terms. Their Universality and Evolution. University of California Press, Berkeley, CA, 1969

16. M. H. Bornstein, "Color vision and color naming: a psychophysiological hypothesis of cultural difference", Psychological Bulletin, 80 (4):257--285, 1973.

17. J. Gage, Colour and Meaning, Thames \& Hudson, 1999

18. T. Belpaeme, "Reaching coherent color categories through communication", B. Kröse et al. (Eds.), Proceedings of the 13th Belgium-Netherlands Conference on Artificial Intelligence (BNAIC'01), Amsterdam, The Netherlands, pp. 41-48, 2001

19. S. Loman, H. Merman: "The KMP: A Tool for Dance/Movement Therapy," American Journal of Dance Therapy, 18 (1), Spring/Summer, 1996.

20. M. Kitajima, K. Don-Han, "Communicating kansei design concept via artifacts: A cognitive scientific approach" Proceedings of the International Workshop on Robot and Human Communication, pp. 321-326, Hakamatsu, Japan IEEE Press, 1998

21. T. Shibata, T. Kato, "Kansei Image Retrieval System for Street Landscape. Discrimination and Graphical Parameters based on correlation of Two Images", IEEE International Conference on Systems Man and Cybernetics, 6, pp. 247252, Tokyo, Japan, 1999

22. K Yoshida, T. Kato, T. Yanoru, “A Study of Database Systems with Kansei Information”, IEEE International Conference on Systems Man and Cybernetics, 6, pp. 253-256, Tokyo, Japan, 1999

23. Y. Isomoto, K. Yoshine, H. Yamasahi, N. Ishi, "Color, Shape and Impression keywords as Attributes of Painting for Information Retrieval", IEEE International Conference on Systems Man and Cybernetics, 6, pp. 257-262, Tokyo, Japan, 1999

24. R. Hattori, M. Fujiyoshi, M. Iida, “An Education System on WWW for Study Color Impression of Art Paintings Applied NetCatalog”, IEEE International Conference on Systems Man and Cybernetics, 6, pp. 218-223, Tokyo, Japan, 1999

25. T. Imai, K. Yamauchi, N. Ishi, "Color Coordination System on Case Based Reasoning System using Neural Networks", IEEE International Conference on Systems Man and Cybernetics, 6, pp. 224-229, Tokyo, Japan, 1999

26. T. Nakata, T. Sato and T. Mori, "Expression of Emotion and Intention by Robot Body Movement", Proceedings of Conference of International Autonomous Systems 5 (IAS-5), 1998.

27. R. Inder, N. Bianchi Berthouze, T. Kato "K-DIME: A Software Framework for Kansei Filtering of Internet Material", IEEE International Conference of Systems, Man and Cybernetics, 6, pp.358-363, Tokyo, Japan 1999

28. N. Bianchi-Berthouze, L. Berthouze, "Exploring Kansei in Multimedia Information", International Journal on Kansei Engineering, 2 (2), pp. 1-10, 2001

29. Y. Rui, T.S. Huang, M. Ortega, S. Mehrotra, "Relevance Feedback: A power tool in interactive content-based image retrieval", IEEE Transaction on Circuits and Systems for Video Technology, 8 (5), pp. 644-655, 1998.

30. G. Aggarwal, T.V. Ashwin, S. Ghosal, "An image retrieval system with automatic query modification", IEEE Transactions on Muktimedia, 4 (2), pp. 201-214,2002

31. L. Taycher, M. La Cascia, S. Scaroff, "Image digestion and Relevance Feedback in the ImageRover WWW Search Engines", Proceedings of the Second International Conference on Visual Information Systems, pp. 85-91, San Diego, 1997 
32. A. Vailaya, M.A.T. Figueiredo, A.K.Jain, H.J.Zhang, "Image Classification for Content-Based Indexing, IEEE Transaction on Image Processing, 101), pp.117-130, 2001

33. N. Bianchi-Berthouze, C. Lisetti, "Modeling multimodal expression of users' affective subjective experience", International Journal on User Modeling and User-Adapted Interaction: Special Issue on User Modeling and Adaptation in Affective Computing, 12 (1), pp.49-84, 2002

34. N. Bianchi-Berthouze, "Mining Multimedia Subjective Feedback", Journal of Intelligent Information Systems, Special Issue on Multimedia Data Mining, O. Zaiane (Eds):, 19 (1), pp. 43-59, Kluwer, 2002

35. A.H. Munsell, A color Notation, Boston, 1905

36. S. Lehar, "Gestalt Isomorphism and the Primacy of Subjective Conscious Experience: A Gestalt Bubble Model", to be published in Behavioral and Brain Sciences, Cambridge University Press, 2002

37. T. Kurita, T. Kato, "Learning of personal visual impression for image database systems", Proceedings of the Second International Conference on Document Analysis and Recognition, pp. 547-552, Tsukuba Science City, 1993.

38. J. Vendrig, M.Worring, A. Smeulders, "Filter Image Browsing: Interactive Image Retrieval by Usig Database Overview, Multimedia Tools and Applications, 15, pp. 83-103, Kluwer Academic Publishers, Netherlands, 2001

39. H. Pashler, "Attention and Visual Perception: Analysing Divided Attention", Visual Cognition, S.M Kosslyn, D.N. Osherson (Eds), 2, pp. 71-100, MIT Press, 1996

40. N. Bianchi-Berthouze, T. Hayashi, "Interpretation of complex data: requirements to support kansei mining", Proceeding of International workshop on multimedia data mining, in conjunction with the Seventh ACM SIGKDD International Conference on Knowledge Discovery \& Data Mining, Edmonton, Canada, 2002

41. H. Kobayashi, "The semantic network of Kansei words", International Conference on Systems, Men and Cybernetics, Nashville, pp. 690-694, Tennessee, 2000

42. N. Bianchi, P. Bottoni, P. Mussio, G. Rezzonico, MG. Strepparava, "Participatory Interface Design: from Naïve Models to Systems", International Conference. on Human Computer Interaction, pp. 24-29, 1997

43. http://www.altavista.com

44. http://www.google.com

45. http://www.yahoo.com

46. N. Miyake, "Making internal process external for constructive collaboration", Cognitive Technology, J. Marsh, C. Nehaniv, B. Gorayska (Eds.), pp. 119-123, IEEE Computer Society, 1997

47. K. Nakakoji, Y. Yamamoto, "Computational Support for Collective Creativity", Knowledge-Based Systems, 13, pp. $451-458,2000$

48. P. Gardenfors, Conceptual spaces: the geometry of thought, MIT Press, 2000

49. E. Tolman, "Cognitive maps in rats and men", Psychological Review, 55, pp.189-208, 1948

50. A. Gopnik, C. Glymour, D. Sobel, "Casual mas and Bayes nets: a cognitive and computational account of theory formation". International congress on Logic, Methodology and Phylosophy of Science, 1999

51. C. Eden, "On the nature of cognitive maps", Journal of Management Studies, 29, pp. 261-265, 1992 